\title{
Index rerum ad vol. 15
}

Acid phosphatase in carrageenin granuloma, 355

Age and function (B), 375

Alopecia (B), 374

Altern (B), 319, 374

Amino acid incorporation by liver micro-somes, 52

Animal in gerontological research (B), 320

Arthus-Reaction, 265

Arylsuphatase-B in carrageenin granuloma, 355

Behavior and experience (B), 375

Biosynthesis of elastin, 85

Blood chemistry, 275

Blutersatz (B), 64

Bone loss and senescence, 140, 155, 171

- $\quad$ marrow and ageing, 155

Calcification of skin, 347

Carbon tetrachloride and hepatic lesions, 7

Caries, 203

Carrageenin granuloma and enzyme activities, 355

Cattle, ligamentum nuchae, 241

Cell nuclei of tail tendon, 258

Chemical composition of ageing tissue, 101

Choice and ageing (B), 240

Chromatin of liver and kidney, 321

Chronic lung disease and ageing of collagen fibres, 24

Collagen, 24, 65, 77, 174, 217, 233, 366

- $\quad$ fibres and lipid, 217

-and lung disease, 24

-, thyroid and food intake, 366

-, molecular crosslinks, 65, 77, 233 Connective tissue of rat kidney, 252 Crosslinks of collagen, $65,77,233$

- $\quad$ of elastin, 85

Degenerative disease, caries, 203

Dental caries, 203

Dietetics (B), 240

Disease (B), 317

DNA conent of tail tendon, 258

Dying and collagen fibres, 24

Elasticity of skin, 121

Elastin, biosynthesis, 85 
Electrolyte balance, extracellular, 31

Exercise and lipid levels in plasma and

tissue, 14 Experience and behavior (B), 375 Extracellular electrolyte balance, 31

Female maturation, 328

Food intake and collagen fibers, 366

Geriatrie (B), 64

Gérontologie (B), 374

Granuloma and enzyme activities, 355

Growth (B), 317

Hematology, 275

Histone from rat liver, 293

Human, age-correlations, 332

blood chemistry, 275

bone, 140, 155, 171

collagen crosslinks, 77

female maturation, 328

380

Index rerum ad vol. 15

intervertebral disc, 174, 189

skin and elasticity, 121

Immunmorphologie bei Arthus-Reaktion,

265 Intervertebral disc, 174, 189

Laboratory animal (B), 320 Learning and rats, 1, 302 Lebistes, tail regeneration, 248

Ligamentum nuchae, swelling and solubility, 241 Lipid and collagen fibres, 217

- $\quad$ levels in plasma and tissue and exercise,

14

Liver chromatin, 321

lesions and carbon tetrachloride, 7

microsomes and amino acid incorporation, 52

- $\quad$ nucleoprotein, 293

Longevità e vitalità (B), 240

Lung disease and collagen fibres, 24

Macromolecules and neuron (B), 374 Maturation of females, 328 Metabolism (B), 376

Microsomes of rat liver and amino acid incorporation, 52 Molecular ageing, 308

- $\quad$ crosslinks of collagen, 65, 77, 233

Mucopolysaccharides of rat kidney, 252

Neuron and macromolecules (B), 374 Noise and ageing (B), 240 Nuclei of tail tendon, 258

Nucleoprotein from rat liver, 293 Nutrition (B), 240

Parental age and female maturation, 328 Plasma and tissue lipid levels and exercise, 14

- $\quad$ volume in rats, 273

Probleme des Alterns (B), 319

Protein composition of the intervertebral

disc, 174, 189 Proteins of blood, 275

Rat, Arthus-reaction, 265

calcification of skin, 347 
granuloma and enzyme activities, 355

hepatic lesions and carbon tetrachloride, 7

and learning, 1, 302

lipid levels and exercise, 14

liver and kidney chromatin, 321

-microsomes and amino acid incorporation, 52 -, nucleoprotein, 293

plasma volume, 273

renal connective tissue, 252

tail tendon, cell nuclei and DNA content, 258

Regeneration of the tail of Lebistes, 248 Renal chromatin, 321

- $\quad$ connective tissue of rats, 252

Retirement process (B), 320

Senescence and bone loss, 140, 155, 171

and molecular ageing, 308 Skin calcification, 347

elasticity, 121

Social learning and rats, 1 Solubility of ligamentum nuchae, 241 Sulphokinase in carrageenin granuloma, 355 Swelling of ligamentum nuchae, 241

Tail regeneration of Lebistes, 248 Temperature and tail regeneration of Lebistes, 248 Tendon, cell nuclei and DNA content, 258 Tissue, age-characteristics, 101

- and plasma lipid levels and exercise, 14

Thyroid and collagen fibers, 366

Vitalità e longevità (B), 240 